\title{
Neige et glaciers dans les Alpes italiennes : un siècle de recherche
}

\author{
L. Mercalli \\ Société Météorologique Subalpine, Turin \\ G. Mortara \\ Consiglio Nazionale delle Ricerche, Istituto Protezione Idrogeological del bacino padano, Turin \\ G. C. Rossi \\ ENEL-CRIS/UII, Venezia-Mestre \\ C. Smiraglia \\ Université de Milan / Comité Scientifique du Club Alpin Italien, Milan \\ Tous les auteurs font partie du Comité Glaciologique Italien
}

\section{PHYSIQUE ET CLIMATOLOGIE DE LA NEIGE}

Les premiers travaux concernant les caractéristiques physiques de la neige dans les Alpes, ont été précédés de l'étude de Donato Rossetti [1] qui, entre les années 1675 et 1681 , réalisa à Turin une recherche sur les structures géométriques des cristaux de neige observés au microscope. Toujours à Turin, commencent en 1780 les premières mesures journalières régulières des hauteurs de neige; cette série est probablement aujourd'hui la plus longue du monde [2]. En 1818 on commence l'activité de mesure de la neige dans une importante station de haute montagne $(2469 \mathrm{~m})$, l'observatoire du Col du Grand SaintBernard [3]. Elle est suivie en 1840 par la ville d'Aoste, dont la série pluvionivométrique, même si c'est une des plus anciennes des Alpes, n'a jamais été étudiée de façon satisfaisante. En 1866 le météorologue barnabite père Francesco Denza établit à Moncalieri, près de Turin, l'Observatoire Météorologique du Real Collegio Carlo Alberto. Outre le fait de créer une série de 130 années de chutes de neige journalières, l'Observatoire de Moncalieri devient le siège de la Corrispondenza Meteorologica Alpino Appennina et le premier réseau italien concernant le milieu montagnard. Grâce à la collaboration avec le Club Alpin Italien et avec les structures religieuses, père Denza installe, entre 1872 et 1890 , plus de 200 stations météorologiques de mesure de la neige fraîche et de la durée de l'enneigement avec notamment la station du Col de Valdobbia (2 $480 \mathrm{~m}$ ) au Sud du Mont Rose, Balme (1 $500 \mathrm{~m}$ ) en aval du massif Albaron-Ciamarella, Cogne (1 $500 \mathrm{~m})$ en Grand Paradis et Crissolo (1 $400 \mathrm{~m})$ pied du Viso. Quelques monographies concernant le climat alpin [4] et les résumés mensuels des données sont régulièrement publiés jusqu'au 1908 dans le Bullettino Meteorologico Mensuale del Real Collegio Carlo Alberto, un exemple d'exceptionnelle organisation pour les moyens de l'époque. Une grande partie des données journalières est malheureuse- ment disparue et les résumés mensuels sont encore à élaborer avec des méthodes modernes. Après la mort de Denza, en 1894, les études et les observations des données nivométriques se réduisent et beaucoup de stations sont fermées. Les premières recherches régionales concernant le phénomène nival sont effectuées en 1887 dans les Alpes orientales par Richter [5] et par Tellini [6] qui, en 1908, publie un important travail sur la distribution de la neige avec 4 cartes (échelle : $1: 750000$ ) fondées sur l'examen de 300 stations italiennes, suisses et autrichiennes. Quelques années plus tard les études sur la climatologie de la neige sont reprises par Monti [7, 8].

La constitution en 1907 de l'Ufficio Idrografico del Magistrato alle Acque de Venise, est suivie en 1913 par l'Ufficio Idrografico del Po dont le siège est à Parma. Cette structure, depuis 1918 Servizio Idrografico Nazionale del Ministero dei Lavori Pubblici, constituera le plus important réseau d'observation pluvionivométrique en Italie. En 1927 les stations nivométriques (mesure journalière des hauteurs de la neige au sol) seront au nombre de 386 pour les Alpes, et 556 en 1950 [9]. L'étude de la neige et de la mesure de son équivalent en eau prend d'année en année une importance majeure dans le cadre de la gestion des usines hydroélectriques en cours de construction $[10,11]$; au contraire les études climatologiques concernant la durée du manteau neigeux recommencent, encore une fois dans les Alpes Orientales, avec les travaux de l'autrichien Kossinna [12] qui publie une carte échelle 1: 1000000 fondée sur les données de 693 stations. En 1944 Gregorig [13] aborde le sujet de l'estimation des flux pluviométriques dans les bassins versants en rapport avec les ressources hydroélectriques, en proposant un modèle pour le calcul du coefficient nivométrique fondé sur la température moyenne de l'air. Avec le travail de Bossolasco [14] concernant les précipitations neigeuses dans I'Italie du Nord, publié en 1948 , la climatologie de la neige italienne voit une des œuvres de plus grande importance, tant par la grande superficie considérée que pour la formulation de relations 
mathématiques destinées à la description de la variation de l'enneigement avec l'altitude, sujet repris en 1950 par Dona [15], seulement pour la région vénétienne, en considérant 236 stations sur la période 1923-44, plus tard étendue à la période 1922-50 [16]. L'intérêt pour les études concernant la neige poussa le Comitato Glaciologico Italiano à créer, en 1950, la Commissione Italiana per lo studio della Neve, soutenue financièrement par l'association des entreprises productrices d'énergie électrique (ANIDEL). Cette commission créa dans les Alpes et les Apennins un petit nombre de stations pilotes, généralement situées près des barrages de haute altitude, destinées à rassembler pendant toute la période d'enneigement les données météo-nivométriques, y compris la densité de la couche de neige, dans le but de rechercher les corrélations avec les débits printaniers. Cette activité fut conduite avec soin et continuité, mais la publication régulière des données dans le Bollettino du Comitato Glaciologico Italiano surtout sous la houlette de Vanni se limita à une phase d'observation et d'application dans le seul but de l'évaluation de la disponibilité de ressources en eau pour la production électrique. Elle fut reprise par Tonini [17]. La vivacité des études nivologiques de la moitié des années 50 amena Passamonti [18] à souhaiter la création en Italie d'un vrai institut spécialisé dans l'étude de la neige, qui malheureusement ne sera jamais constitué. Tandis qu'on commence à recueillir le fruit de l'activité de la Commissione Nevi du CGI dans les comptes rendus de Vanni [19] et Morandini [20-21], Bossolasco établit l'analyse de problèmes de physique de la neige [22-23, 24], jusqu'alors peu étudiés et Capello [25] ouvre une intense phase de recherche sur l'enneigement des Alpes occidentales. En 1961 Drigo publie les premiers résultats sur l'emploi des méthodes radioactives pour la mesure de l'équivalent en eau de la neige [26]. Malheureusement ces recherches sont vite abandonnées et pendant plus de trente ans on continuera à faire confiance seulement sur les traditionnelles mesures gravimétriques. En 1989 le problème de l'insuffisante fiabilité des mesures d'équivalent en eau de la neige par fusion dans le pluviomètre, déjà connu depuis longtemps [27], est relevé par Mercalli [28] sur la base de vingt ans de mesures dans l'Alta Ossola ; en 1992 Capra et al. [29] abordent de nouveau le problème de la mesure de l'équivalent en eau de la couche de neige en exploitant la radioactivité naturelle.

Entre-temps les premiers bilans de plus de cinquante ans de travail sont proposés en 1961 et 1968 par Zanon [30, 31]. En 1962, C. Capello de l'institut de géographie alpine de l'université de Turin apporte un nouveau souffle à l'étude de la climatologie nivale et la prévision des avalanches en Italie. Le premier travail produit par l'Istituto turinois est l'importante bibliographie de Gili-Borghet [32] qui relate l'ensemble des travaux concernant la neige publié en Italie depuis leurs origines jusqu'en 1950 ; suivront d'autres études de climatologie de la neige soit seulement sur un cycle d'enneigement soit des périodes plus longues. Nous citerons les travaux de Bonanate [33] en Veneto et de Ogliaro [34] en Piémont. Malheureusement cette structure de recherche réduira son activité pour disparaître à la fin des années 70 , probablement parce que, née dans le domaine universitaire des facultés de sciences humanistes, elle n'eut pas la possibilité d'évoluer vers des sièges scientifiques plus développés qui de toute façon en ignorent leur activité.
En 1973 le Servizio Idrografico du Ministère des Travaux Publics présente une analyse détaillée concernant l'enneigement en Italie pendant la période 1921-1960 [35]. Ce document très complet et unique, trace l'histoire de la nivologie et fournit des résultats au niveau national. Les conclusions de ce rapport sont toutefois négatives, en mettant en évidence que depuis 1970 l'intérêt pour les études physiques et climatologiques concernant la neige subit une atténuation. Beaucoup de sujets sont encore à étudier, les recherches sont nombreuses mais dispersées et sans aucune coordination. Il manque aujourd'hui un institut qui traite de programmes de recherches structurés et modernes. Malheureusement, après 20 ans de la publication de l'étude du Servizio Idrografico, la situation n'est pas changée. En effet, bien que l'intérêt pour le problème des avalanches soit croissant comme on verra après pour le Club Alpin Italien et l'AINEVA et pour l'Armée Italienne, les expériences scientifiques concernant la neige ne progressent pas, et les quelques recherches en cette période ont un caractère local sans trait cohérent avec les besoins du territoire. Il manque un groupe de recherche engagé dans le secteur nivologique, ce qui a un résultat négatif ; la formation technique et scientifique du personnel est partielle aujourd'hui, et se fonde sur différents cours universitaires - géologie, sciences forestières, sciences naturelles - sans que des liaisons avec les physiciens et les ingénieurs ne se développent vraiment. Récemment des nouveaux réseaux d'observation des paramètres nivométriques, en télétransmission, ont été installés dans toutes les Alpes, en concertation avec différents organismes régionaux.

Malgré un essai de coordination avec l'AINEVA, nous croyons que soit encore plus nécessaire la présence d'un institut supérieur de recherche à même de fournir les informations précises pour la meilleure utilisation de moyens et personnel. Outre les nouvelles investigations, il existe un patrimoine considérable de données du passé à utiliser et élaborer avec les méthodes modernes. Dans le tableau $I$ on indique l'actuelle composition du réseau « historique » de stations nivométriques du Servizio Idrografico Italiano dans le secteur alpin.

Il s'agit d'environ 600 stations dont 165 mesurent en continu depuis plus de 70 ans les hauteurs journalières de neige au sol; plus d'une centaine ont un historique de 50 ans. La plus grande partie de ces données n'a jamais été publiée (dans les Annali Idrologici il y a seulement des résumés mensuels et rarement les données journalières). Compte tenu de l'absence de longues séries de données nivométriques, surtout en haute montagne, il serait opportun de sauvegarder les archives manuscriptes, en les transférant sur ordinateur et en avançant dans l'élaboration de statistiques territoriales numériques et cartographiques. Ce travail profiterait aux études sur le changement climatique, à la gestion des ressources hydriques et aux activités touristiques hivernales. Récemment nous avons commencé l'informatisation de quelques séries historiques de hauteur de la neige surtout dans les Alpes occidentales, avec la formation d'une banque de données journalière sur ordinateur [36, 37, 38, 39]. Il s'agit toutefois d'une tâche encore trop limitée qui devrait trouver dans le Servizio Idrografico Italiano les instruments pour évoluer dans un projet organique et global qui garantisse le fonctionnement des stations plus anciennes dont beaucoup à l'heure actuelle risquent de fermer. 
Tableau 1. - Stations pluvionivométriques du Servizio Idrografico Italiano dans les Alpes, par tranches d'altitude (m) et longueur de la série d'observation (années).

\begin{tabular}{|c|c|c|c|c|c|c|c|c|c|c|c|c|}
\hline Compartimento & $\mathrm{km}^{2}$ & N. stations & $\begin{array}{l}\mathrm{km}^{2} \\
\text { /staz. }\end{array}$ & $\begin{array}{c}0-500 \\
\mathrm{~m}\end{array}$ & $\begin{array}{c}501-1000 \\
m\end{array}$ & $\begin{array}{c}1001-1500 \\
\mathrm{~m}\end{array}$ & $\begin{array}{c}1501-2000 \\
m\end{array}$ & $>\underset{\mathrm{m}}{2000}$ & $\begin{array}{c}>70 \\
\text { années }\end{array}$ & $69-60 a$ & $59-50 a$ & $<50 a$ \\
\hline Venezia & 36760 & 390 & 94 & 198 & 91 & 79 & 20 & 2 & 65 & 238 & 75 & 12 \\
\hline Milano & 30400 & 101 & 301 & 20 & 37 & 23 & 12 & 9 & 44 & 45 & 10 & 2 \\
\hline Torino & 25200 & 104 & 245 & 11 & 35 & 31 & 18 & 9 & 56 & 13 & 20 & 15 \\
\hline Total & 92360 & 595 & 155 & 229 & 163 & 133 & 50 & 20 & 165 & 296 & 105 & 29 \\
\hline
\end{tabular}

\section{AVALANCHES}

Les études concernant les avalanches en Italie fonctionnent depuis longtemps en parallèle avec les recherches traitant de la climatologie de la neige depuis le $\mathrm{XVI}^{\mathrm{e}}$ siècle. Un important patrimoine de chroniques d'avalanches et de leurs dégâts est conservé en différentes archives municipales et régionales. Seulement une petite fraction a été analysée. Les premières études détaillées sur les avalanches sont datées de 1755 avec la description par le médecin de la maison de Savoie Somis [40] de l'avalanche de Bergemoletto (Valle Stura, Cuneo). Avec la fondation à Turin du Club Alpin Italien, en 1863, une nouvelle source d'informations descriptives concernant les avalanches est décrite par le Bollettino del Cai, qui plus tard s'enrichira de l'œuvre de Denza. Cela rappelle d'ailleurs l'importante étude sur les avalanches pendant les hivers 1885 et 1888 [41]. Après une phase de déclin, les études recommencent pendant les années 20 avec Valbusa [42, 43] qui a le mérite de poser les premiers problèmes à un niveau de physique appliquée ; personne ne suivra ce modèle de recherche. On revient donc à une méthode descriptive dans le strict respect du travail antérieur. Cette activité s'exprime surtout à l'Institut de Géographie Alpine de Turin, avec les nombreuses études de Capello, entre 1967 et 1975 $[44,45,46]$, transcrites dans l'atlas des avalanches des provinces de Turin et Cuneo [47, 48]. Après la fermeture de l'Institut de Géographie Alpine, arrive une période de production scientifique limitée, seuls quelques auteurs continuent à effectuer des recherches à caractère exhaustif et rigoureux sur l'histoire des avalanches : c'est le cas des deux travaux de Fontana concernant la Valsesia [49, 50]. En 1967 le CAI institue un Service Avalanches qui est chargé de la sécurité des touristes et des professionnels : s'ouvre alors une phase d'application conduite avec des instruments modestes. Dans les années 70, le IV Corpo d'Armata Alpino, sensible aux problèmes de la sécurité des détachements, fonde le service METEOMONT, qui installe ses propres stations de mesure et commence un long travail de cartographie des avalanches dans l'arc alpin (échelle 1: 25000 ). Au cours des années 80 , tandis que le Service Avalanches du CAI s'occupe activement de la formation professionnelle et didactique, les régions italiennes de l'arc alpin créent des services de prévision du risque d'avalanches, fondés d'une part sur stations de mesures existantes, d'autre part sur de nouveaux réseaux automatiques; en 1983, ces services se fédèrent au sein de l'AINEVA (Associazione Interregionale per lo Studio della Neve e delle Valanghe), dont le siège est à Bormio. Aujourd'hui l'AINEVA, avec sa revue « Neve e Valanghe», est deve- nue la principale structure de recherches concernant les avalanches des Alpes italiennes. Elle a le mérite d'avoir favorisé une standardisation des méthodes de mesure et de diffusion des bulletins. On observe d'autre part que l'activité des Services Avalanches italiens se préoccupe seulement de l'aspect «terrain " lié à des méthodologies plutôt traditionnelles. Il manque ainsi une unité de recherche universitaire constituée par physiciens et ingénieurs chargés de développer de nouveaux modèles pour l'analyse des phénomènes de la neige.

\section{GLACIOLOGIE}

Dans les Alpes italiennes il y a aujourd'hui un millier de glaciers qui couvrent une surface de $600 \mathrm{~km}^{2}$ env. Les premières observations descriptives remontent à la deuxième moitié du XVIII ${ }^{\mathrm{e}}$ et concernent surtout les glaciers du Mont-Blanc et de Savoie, qui en ce temps-là appartenaient au Royaume de Sardaigne ; nous rappelons l'œuvre de Bourrit [1,2] et de Saussure [3]. A partir de 1680, beaucoup de cartographies des Alpes occidentales ont été réalisées par le Stato Maggiore Generale Sardo alors que le gouvernement autrichien s'intéressait à la région Lombardie-Vénétie. Durant la première moitié du XIX ${ }^{\mathrm{c}}$, et précisément pendant les phases les plus importantes du Petit Age de Glace, on découvre les travaux de Parrot [5], Welden [6] et des frères Schlagintweit [7] concernant les glaciers du Mont-Rose. Ils sont suivis des études de King [8] sur le Valpelline et des monographies détaillées de Baretti sur les glaciers du Ruitor [9], de la Brenva [10] et du Miage [11]. Pour le secteur centre oriental il s'agit des études de Moisisovics [12], Grohmann [13], du topographe Payer [14] des géographes Stoppani [15] et Richter [16]. Il faut rappeler dans le même temps le travail du Club Alpin Italien (CAI), créé à Turin en 1863, qui publie dans son Bollettino beaucoup de rapports, souvent avec documentation photographique de qualité exceptionnelle (comme celle de V. Sella). La prévoyance du CAI de deviner l'importance de l'étude des glaciers se réalise en 1895 avec la constitution de la Commissione Glaciologica Italiana. Cette commission a le mérite de mettre en train les premières campagnes annuelles sur le contrôle des déplacements des fronts des glaciers et de promouvoir des recherches géographiques approfondies d'exploration dans la plupart des Alpes. On rappelle pour le secteur occidental, le travail de Viglino [17], Mader [18] et Roccati [19] concernant les Alpes Maritimes, Druetti \& Porro [20] et Monti [21] sur le Grand Paradis, de Revelli [22] sur le Mont-Blanc, de Dainelli [23] dans le Mont-Rose ; pour les 
Alpes centrales Marson [24] et Sangiorgi [25] sur le Bernina-Disgrazia, Mariani [26] sur Ortles-Cevedale ; pour les Alpes orientales la magnifique monographie de 1913 de Finsterwalder [27] concernant le glacier de Solda, les travaux de Toniolo [28] sur la Marmolada et de Bonfioli [29] en Trentino et la vaste production par Marinelli G. [30] et Marinelli O. [31]. Dans cette zone on appréhende mieux la dynamique glaciaire pendant les combats de la première guerre mondiale ; en effet quelques glaciers à la frontière furent traversés par environ $10 \mathrm{~km}$ de tunnels qui arrivèrent jusqu'au sommet de l'Ortles à $3902 \mathrm{~m}$ [32]. La commission glaciologique, malgré un nombre relativement faible de chercheurs par rapport à l'étendue des surfaces glaciaires, posa les jalons pour la constitution d'un patrimoine de connaissances scientifiques qui aujourd'hui est encore le plus important au monde. En 1913 fut fondé à Turin le Comité Glaciologique Italien (CGI), organisme scientifique qui hérita de la remarquable activité de l'ancienne commission en donnant une nouvelle orientation à l'étude des glaciers. Les résultats de ces recherches qui concernent toutes disciplines liées à la glaciologie, sont publiés, à partir de 1914, dans le Bollettino del Comitato Glaciologico Italiano qui compte aujourd'hui 66 volumes. Une section du bulletin est consacrée depuis 1927 aux rapports concernant les observations annuelles des fronts de centaines de glaciers, quelques-uns mesurés depuis 80 ans (Brenva, Lys, Ventina). Le corpus de ces rapports constitue un document de plus de 1500 pages et devient la base pour n'importe quel examen des variations glaciaires en Italie, surtout grâce aux nombreuses photographies qui sont aujourd'hui des documents irremplaçables. Sous l'égide du CGI et en collaboration avec des services techniques d'Etat (Servizio Idrografico, Istituto Geografico Militare), entre 1925 et 1927 prennent naissance des projets importants (I'Elenco dei Ghiacciai italiani [33] et l'Atlante dei ghiacciai italiani [34]), qui représentent les premiers exemples dans le monde de dénombrement systématique de glaciers. Durant 50 ans on publie dans le Bollettino del CGI et dans d'autres revues, de nombreuses monographies de géomorphologie descriptive concernant les principaux bassins glaciaires. Federico Sacco apporte des connaissances fondamentales sur la glaciologie italienne en publiant une centaine de travaux, entre lesquels on compte les cartes des grandes moraines quaternaires de la plaine du Pô $[35,36,37,38,39]$, les recherches sur les glaciers du Ruitor [40], du Mont-Blanc [41], du Grand Paradis [42], de Valsesia et Ossola [43], la bibliographie glaciologique italienne du 1927 avec plus de 1000 références [44]. Les glaciers du Grand Paradis sont étudiés pendant plusieurs années par Peretti [45], tandis que ceux du Mont-Rose sont contrôlés soigneusement par Umberto Monterin, pionnier dans la recherche de corrélations entre variations climatiques et glaciaires [46, 47, 48]. En Lombardie Merciai $[49,50]$ décrit les glaciers du massif Adamello-Presanella ; Ardito Desio résume, dans la grande monographie de 1967 [51], plus de 40 ans d'observations sur le bassin glaciaire italien le plus étendu qui, en 1944 - avec $82 \mathrm{~km}^{2}$ - montrait une réduction de surface de $33 \%$ par rapport aux évaluations du $1888\left(123 \mathrm{~km}^{2}\right)$. Le sujet de la physique de la glace est abordé en 1925 par Somigliana, qui formula une théorie du mouvement glaciaire pour la détermination de l'épaisseur des glaciers [52], vérifiée ensuite par la campagne de forages dans le glacier de l'Hohsand conduite en 1943 par Calciati [53]. Ce sujet, après les études de Aliverti [54], Udeschini [55] et Agostinelli [56], ne se développera plus. La collaboration du CGI avec le Servizio Idrografico, débutée en 1917 avec les études sur l'hydrologie glaciaire dans le bassin du Lys par Giandotti [57], se renforce entre 1930 et 1960 suite à l'intérêt croissant pour l'exploitation des ressources hydroélectriques. A ce propos on rappelle les recherches par Alfieri $[58,59]$ et Vanni $[60]$ dans les Alpes occidentales, de Visentini [61] et Abbadessa [61] sur le Glacier des Forni, le plus étendu des Alpes italiennes $\left(13 \mathrm{~km}^{2}\right)$. La participation du CGI aux activités organisées pour l'Année Géophysique Internationale 1957-58 se concrétise avec la réalisation du Catasto dei Ghiacciai Italiani [63], coordonné par le géographe G. Nangeroni. Malgré d'innévitables imprécisions causées par la cartographie disponible en ce temps-là, l'œuvre en quatre volumes énumère les caractéristiques de tous les glaciers italiens et elle représente aujourd'hui encore un moyen valable de connaissance. L'initiative internationale encourage aussi les recherches dans le domaine géophysique qui, commencées en 1953 avec les mesures d'épaisseur du glacier de la Marmolada [64] et des Forni [65], continuent jusqu'en 1972 dans les plus importants glaciers italiens $[65,66,67$, $68,69,70]$. En cette période, surtout sous l'inspiration de Lesca [71, 72, 73], s'ouvre aussi une féconde production cartographique à grande échelle qui reprend les premières expériences de 1920 et 1925 sur le glacier du Lys [74]. II ne faut pas oublier les études concernant les relations climat-glaciers, à la suite de recherches palénologiques dans la tourbière du Ruitor [75] et lichenométriques dans les Alpes centrales [76]. Après le 1965, avec l'institution de l'Ente Nazionale per l'Energia Elettrica (ENEL), l'objectif des études appliquées se porte sur l'évaluation des ressources hydriques pour la production d'énergie ; débutent alors les mesures de bilan de masse sur le glacier du Caresér (Alpes centre orientales) qui, sous la coordination de Zanon [77], se poursuivent encore aujourd'hui et constituent la plus longue série pour le côté Sud des Alpes. Dans les années 70, comme le fait observer Smiraglia [78], l'évolution positive de la glaciologie italienne s'arrête. La réduction sensible des travaux à caractère glaciologique entraîne une profonde crise structurelle de ce secteur scientifique. Même si on compte des travaux remarquables, comme ceux de Belloni concernant le bilan hydrologique de bassins glaciaires [79], de Belloni [80] et Cerutti [81] concernant les rapports climat-glaciers, de Rabagliati \& Serandrei-Barbero [82] sur l'emploi du remote-sensing de satellite pour le contrôle des glaciers, et la bibliographie analytique de Pantaleo [83] avec 8400 références, la phase de déclin se poursuit et emmène en 1978 à la transformation du Bulletin du CGI dans la revue Geografia Fisica e Dinamica Quaternaria, qui publia des sujets sur des thèmes autres que la glaciologie. Dans les 32 fascicules parus entre 1978 et 1993, plus de la moitié ne présente pas des articles de sujet glaciologique, exceptées les campagnes annuelles de mesure. On note que les vastes ouvrages géographiques font défaut, que les secteurs de physique et de géophysique n'ont pas été développés, et que l'intérêt pour la physique et la chimie de la glace n'est pas encore né. Les raisons du faible intérêt pour la glaciologie en Italie peuvent se résumer aux nombres réduits de chercheurs, à l'absence de cours universitaires spécifiques, aux disponibilités financières trop limitées. Toutefois demeurent les campagnes glaciologiques annuelles jamais interrompues depuis 1911 (fig. I), et la collaboration avec le World Glacier Monitoring Service continue. Les initiatives im- 
portantes ne manquent pas : mise à jour du cadastre des glaciers italiens de 1959-61 dans le cadre du World Glacier Inventory (1979-1983), en grande partie réalisé par Secchieri [84] et fondé sur l'interprétation aérophotographique (même si les données analytiques n'ont pas été publiées à ce moment-là) et organisation de congrès glaciologiques de Bolzano (1983) et Gressoney (1991). Depuis quelques années on note une reprise de l'intérêt pour les glaciers, symptôme d'un effort pour se conformer aux développements de la glaciologie des autres Pays :

- les entreprises productrices d'énergie hydroélectrique soutiennent de nouvelles études sur l'évaluation de l'impact des variations climatiques sur les ressources hydriques [85]; en particulier de nouveaux bilans de masse sont mis en marche par l'ENEL dans les Alpes orientales [86] et sur le Mont-Rose [87], par les Aziende Energetiche Municipali de Turin sur le Grand Paradis [88] et de Milan sur l'OrtlesCevedale [89];

- en plus de s'adjoindre le CGI dans les campagnes annuelles de mesure, de nouveaux groupes de travail mis en place par le Club Alpin Italien où des organismes publics locaux apportent leur contribution à des études. Le Servizio Glaciologico Lombardo publie en 1992 le nouveau cadastre des glaciers de la Lombardie [90] ; la Società Alpinisti Tridentini et le Servizio Idrografico de la Province Autonome de Bolzano s'occupent de nouveaux bilans de masse dans les Alpes centre orientales;

- l'écroulement du glacier Coolidge Supérieur sur le Mont Viso en 1989 [91] donne l'occasion à Dutto et al., d'étudier le sujet, presque méconnu en Italie, des risques naturels liés à la dynamique glaciaire [92] ;

- l'approfondissement des recherches concernant les glaciers rocheux par des mesures géophysiques, électriques et radar [93], ainsi que les relations climat et glaciers $[94,95]$;

- le développement des recherches glaciologiques dans les zones polaires, coordonnées par Orombelli $[96,97]$, dans le cadre de la participation italienne aux programmes internationaux ;

- la parution de publications glaciologiques de vulgarisation en réponse au croissant intérêt du public pour les sujets de l'environnement. On rappelle l'œuvre de Zanon [98] sur les glaciers du Veneto, l'histoire des glaciers du Mont-Rose [99], un guide de glaciologie par Smiraglia [100], la revue internationale de climatologie alpine Nimbus éditée par la Société Météorologique Subalpine à Turin.

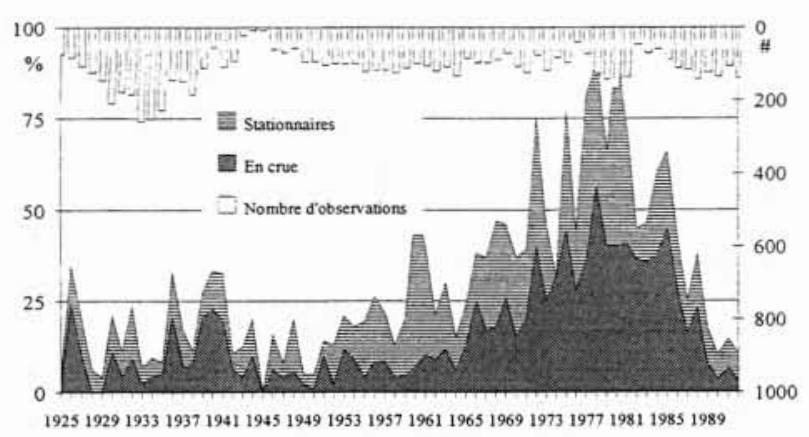

1. Variations du front des glaciers italiens d'après les campagnes glaciologiques du CGI de 1925 à 1992.
La glaciologie italienne part donc d'une solide tradition, mais à son $100^{c}$ anniversaire elle nécessite un fort renouvellement pour rejoindre les plus récents développements internationaux. Pour atteindre cet objectif, il serait souhaitable que s'instaure une réelle collaboration entre les différents partenaires intéressés par ce sujet.

\section{Références}

\section{Neige et avalanches}

[1] Rossetti D. (1681). - La figura della neve. Torino, Bibl. Civ.

[2] Leporati E., Mercall. L. (1993). - La neve a Torino : due secoli di misure. Analisi climatologica e azione sulle strutture. Nimbus, 1.

[3] JANIN B. (1970). - Le Col du Grand-Saint-Bernard. Climat et Variations Climatiques. Impr. Marguerettaz-Musumeci, Aoste.

[4] DENZA F. (1877). - Studi sulla climatologia della Valle d'Aosta. Torino.

[5] RICHTER E. (1887-88). - L'altitudine delle nevi delle Alpi Orientali. Cron. Soc. A. Fr.

[6] TEllini A. (1908). - La distribuzione della neve nelle Alpi Orientali e nel Veneto. Atti R. Ist. Ven. Sc. Lett. Arti, 67.

[7] MonTI V. (1912). - Secondo saggio di nuove ricerche sui ghiacciai del Gran Paradiso. Il limite climatico delle nevi e prime linee della climatologia della zona glaciale del gruppo. Riv. Geog. It.

[8] MonTI V. (1913). - Sulla distribuzione altimetrica della nevosità e dell'ablazione in alta montagna. Sui fondamenti della determinazione del limite della neve. Ann. Uff. Centr. Met. Geodin, 34, Roma.

[9] Gazzolo T. (1964). - Le réseau Hydrométéorologique du Service Hydrographique Italien. Publ. n. 67 AIHS.

[10] Giandotri M. (1919). - Il fenomeno dell'accumulamento e della fusione delle nevi in relazione al regime degli afflussi. L'Energia Elettrica, 5.

[11] Crestani G. (1937). - Sulla determinazione dell'equivalente in acqua e della densità della neve. Ann. LL. PP., $75(6)$.

[12] Kossinna E. (1939). - Die Schneedecke der Ostalpen. Wissen. Veröffent. Deuts. Museum fuer Landerkunde.

[13] GREgorig R. (1944). - Sugli afflussi ai bacini idrografici, con particolare riguardo a quelli alpini. L’Energia Elettrica, 21(11-12).

[14] Bossolasco M. (1948). - Le precipitazioni nevose nell'Italia settentrionale. Geof. Pura e Appl., 12-13.

[15] DONA F. (1950). - Programma di studio e nota preliminare sulla distribuzione altimetrica del manto nevoso e delle precipitazioni nevose nella regione veneta. Atti $25^{\circ}$ Congr. Geogr. Ital., 1, Torino.

[16] DONA F. (1954). - Caratteristiche nivomentriche di alcune località delle Alpi orientali con più lungo periodo di osservazione. Atti $16^{\circ}$ Congr. Geogr. Ital., Padova-Venezia.

[17] ToNINI D. (1951). - Sulla correlazione tra precipitazioni nevose e le portate di un corso d'acqua tipico delle Alpi Orientali. L'Acqua, 5-6.

[18] Passamonti L. (1955). - Necessità della creazione in Italia di un Istituto per lo studio della neve. Geof. e Meteor., 3.

[19] VANNI M. (1955). - Ricerche sul manto nevoso quale riserva di acqua nei rapporti coi deflussi. L'Energia Elettrica, 34(1). 
[20] MORANDINI G., VANNI M. (1958), - Le osservazioni del manto nevoso in Italia nel periodo 1951-52/1955-56. L'Energia Elettrica, 35(8).

[21] MORANDINI G. (1963). - Dieci anni di osservazioni sul manto nevoso in Italia - 1951-52/1960-61. Scritti Geogr. in onore di G. Colamonico, Loffredo, Napoli.

[22] Bossol.asco M. (1954). - Sulla micrometeorologia del manto nevoso. Geof. e Meteorol., 1-2.

[23] Bossol.asco M., Dagnino I. (1957). - Densité de la neige et température de l'air: La Météorologie, 1.

[24] Bossolasco M., Dagnino I. (1958). - Caratteristiche fisico-meteorologiche del manto nevoso. Geofis. e Meteorol., 4-5.

[25] Capello C.F., Luchino M. (1957-58). - Il limite temporaneo delle nevi nelle Alpi occidentali italiane. Boll. Com. Glac. It., 2s, 8 .

[26] Drigo A., Cecchetti A., Fratucello G. (1961). - Misure dell'equivalente in acqua del manto nevoso col metodo dell'assorbimento dei raggi gamma, effettuate alla Fedaia (Marmolada) nel 1959-60 e nel 1960-6I. Boll. Com. Glac. It., 2s., 11(2).

[27] GIANDOTTI M. (1918). - Sulla ricerca delle precipitazioni in alta montagna e sul funzionamento dei pluviometri totalizzatori nel bacino del Po. Min. LL. PP., Uff. Idrog. del Po, Parma.

[28] Mercallu L., Viarengo S. (1989). - Problèmes posés par la mesure de l'équivalent en eau de la neige par des pluviographes chauffés. Publ. Ass. Int. Clim., 2.

[29] Capra D., de Pasquale N., Rossi G. (1992). - Mesure de l'épaisseur équivalente en eau de l'enneigement au moyen d'un spectromètre gamma aérien. Réunion SHF, Sect. Glaciol., 12-13 mars 1992, Grenoble.

[30] Zanon G. (1960-61). - Ricenche sulla neve in Francia e in Svizzera. Osservazioni e prospettive delle ricerche nivometriche in Italia. Boll. Com. Glac. It., 2s., 11.

[31] ZANON G. (1968), - La neve ed alcuni suoi problemi. Monti e Boschi, 19(3).

[32] Gil..-Borghet A. (1967), - Gli studi sulla neve in Italia dalle origini al 1950. Pubbl. Ist. Geog. Alpina, 1, Torino.

[33] BonANATE C.M. (1970), - Il ciclo nivale nel Veneto negli inverni dal 1929-30 al 1958-59. Pubbl. Ist. Geogr. Alpina, 14. Torino.

[34] Ogliaro L. (1971). - Frequenza, permanenza e ciclo della neve in Piemonte nel trentennio 1929-1958. Pubbl. Ist. Geogr. Alpina, 17, Torino.

[35] Min LL. PP. SERVIZIO IDROGRAFICO (1973), - La nevositd in Italia nel quarantennio 1921-1960. Pubbl. n. 26. Ist. Poligr. dello Stato, Roma.

[36] Mercalli L., Brizio D. (1992). - Pioggia e neve a Bra, 130 anni di osservazioni (1862-1991). Quad. Oss. Met. Museo Civ. Craveri di St. Nat, Città di Bra.

[37] Biancotti A., MerCAlli L. (1991). - Variazioni climatiche a breve termine (1927-89) a Gressoney (Valle d'Aosta, Italia), $1850 \mathrm{~m}$ s.l.m. Rev. Valdôtaine Hist. Nat., 45.

[38] Mercall. L., PALudi S. (1993). - La neve nelle Valli di Lanzo: caratteristiche climatologiche e tendenze evolutive. Nimbus 2.

[39] Romano F., MerCalli L. (1994), - L'Osservatorio Meteorologico di Cuneo : dal 1877 sentinella della Granda. Nimbus, 3.

[40] Somis I. (1758). - Ragionamento sopra il fatto avvenuto in Bergemoletto in cui tre donne sepolte fra le rovine della stalla per la caduta di una gran mole di neve sono state trovate vive dopo trentasette giorni. Stamperia Reale, Torino.

[41] DENZA F. (1889). - Le valanghe degli inverni 1885 e 1888. Boll. CAl 22, 55.

[42] VAlBuSA U, (1924), - Il meccanismo interno di avanzata e di arresto della valanga. Atti Congr. S.I. Pr. Sc., Napoli.

[43] VAlBuSA U. (1924). - L'azione della neve nell'atterramento delle linee aeree di montagna. La Meteorol. Pratica, Subiaco.

[44] CaPello C.F. (1967). - Le valanghe in Piemonte negli inverni dal 1957-58 al 1962-63 (con atlante). Ist. Geog. Alpina, Torino.

[45] CAPELLo C.F. (1968), - Le ricerche sulle valanghe in Italia dalle origini al 1965. Ist. Geog. Alpina, 9, Torino.

[46] CAPELLO C.F. (1974), - Ricerche e studi sulla valanga del M. Crammont. Ist. Geog. Alpina, Torino.

[47] CAPELlo C.F. (1977), - Archivio storico topografico delle valanghe italiane. Provincia di Cuneo. Amm. Prov. di Cuneo/Ist. Geog. Alp., 1.

[48] CaPello C.F. (1978), - Archivio storico topografico delle valanghe italiane. Provincia di Torino. Amm. Prov. di Torino / Ist. Geog. Alp., 2.

[49] FonTANA E. (1983). - Inverni valsesiani. Ed. Corradini, Borgosesia.

[50] FontanA E. (1991). - Storie di antichi inverni. CA1, Varallo Sesia.

\section{Glaciers}

[1] Bourrit M.T. (1776), - Description des aspects du MontBlanc du côté de la Val d'Aoste. Genève.

[2] Bourrit M.T. (1785). - Nouvelle description générale et particulière des glacières, vallées de glace et glaciers qui forment la grande chaîne des Alpes de Suisse, d'Italie, et de Savoie. Genève.

[3] DE SAussure H.B. (1796), - Voyages dans les Alpes. 4 vol., Neuchâtel.

[4] ANich P., Hueber B. (1774). - Atlas Tyrolensis. Wien.

[5] PARROT F. (1817). - Beobachtungen u. die Scheneegrenze u. die Höhenverhaltnisse auf der Sudseite des Monte Rosa. Schweiger's Journal, 19.

[6] Welden F. (1824). - Der Monte Rosa. Eine topographische und naturhistorische Skizze. Wien.

[7] Schlagintweigt A., Schlagintweigt H. (1850), - Untersuchungen über die physicalische geographie er Alpen in ihren Bezichung zur den phenomenen der Gletscher, zur Geologie Meteorologie und Pflanzengeographie. Leipzig.

[8] KING S.W. (1858), - The Italian Valleys of the Pennine Alps. Murray Ed., London.

[9] BARETTI M. (1880). - Il lago del Rutor. Boll. Club Alpino It., 14.

[10] BARETTI M. (1880), - Il ghiacciaio del Miage. Versante italiano del gruppo del Monte Bianco. Mem. R. Acc. Sc. di Torino, ser. 2, 32.

[11] BARETTI M. (1881). - Monografia del Ghiacciaio della Brenva. Boll. Club Alpino It., 45.

[12] Moisısovicz E. (1863), - Die alten Gletscher der Südalpen. Mitl. d. Oe. Alpen Vereins.

[13] Grohmann L. (1863). - Die Vedretta Marmolada. Mittl. d. Oe. Alpen Vereins. 
[14] PAYER J. (1865). - Die Adamello-Presanella Alpen. Peterm. Mitteil., 17.

[15] Stoppani A. (1880), - Geologia d'Italia. Parte II : l'era neozoica. Ed. Vallardi, Milano.

[16] RICHTER E. (1888). - Die Gletscher der Ostalpen. Handbucher zur Deutsch. Landes und Volskunde, 3.

[17] VIgLINo A. (1898). - Introduzione allo studio sui ghiacciai delle Alpi Marittime. Boll. Club Alp. It., 31.

[18] MADER F. (1908). - Appunti sui ghiacciai delle Alpi Marittime. Riv. Mens. Club Alp. It., 28.

[19] RocCATI A. (1912). - I ghiacciai del gruppo Clapier, Maledía, Gelas. Riv. Mens. Club Alpino It., 31.

[20] Druetti A., Porro F. (1895-96). - Osservazioni sui ghiacciai del Gruppo del Gran Paradiso. Contributo allo studio dei movimenti dei ghiacciai. Boll. Club Alp. It., 29.

[21] MonTI V. (1908), - Primo saggio di nuove ricerche sui ghiacciai del Gran Paradiso. Annali Uff. Centr. Met. Geod., 32, p. I.

[22] ReVelli P. (1912). - Le fronti di sette ghiacciai nel versante italiano del Monte Bianco nel 1911. Riv. Mens. Club Alp. It., 31.

[23] Dainelli G. (1906). - Alcune notizie sopra $i$ Ghiacciai delle Valli di Gressoney e d'Ayas. Zeitschr. Gletscherkunde, I.

[24] MARSON L. (1896), - Sui ghiacciai del Massiccio del Monte Disgrazia o Pizzo Bello. Boll. Soc. Geogr. It., 6.

[25] SANGIORgI D. (1912). - Osservazioni sui ghiacciai italiani del gruppo del Bernina e del Disgrazia. Riv. Mens. Club Alp. It., 31.

[26] MARIANI E. (1905). - Osservazioni su recenti oscillazioni di alcuni ghiacciai del Gruppo Ortles-Cevedale. R. Ist. Lomb. Sc. Lett., 38.

[27] Finsterwalder S., Magally M. (1913). - Die Nuevermessung des Suldenferners 1906 und dessen Veränderunggen in den letzen Jahrzehnten. Zeitschr. Gletscherkunde, 3 , helft 3 .

[28] Toniolo R. (1907), - Osservazioni e riscontri sui ghiacciai del gruppo della Marmolada. Riv. Fis. Mat. Sc. Nat., 8.

[29] BONFIOLI A. (1911). - La misurazione dei ghiacciai trentini. Boll. SAT, 6.

[30] Marinelli G. (1899). - Lo studio dei movimenti dei ghiacciai italiani nel biennio 1897-98. Riv. Geogr. It., 5-6.

[31] MARINELli O. (1910). - I ghiacciai delle Alpi Venete. Riv. Mens. Club Alpino It., 29.

[32] KLEBELSBERG R. (1920]. - Glazialgeologische Erfahrungen aus Gletscherstollen. Zeitschr. Gletscherkunde, 9.

[33] PORRo C. (1925). - Elenco dei ghiacciai italiani. Ufficio Idrogr. del Po.

[34] PORRo C., LABIUS P. (1927). - Atlante dei ghiacciai Italiani. Carta Corografica I: 500000 . IGM, Firenze.

[35] SACCO F. (1887), - L'Anfiteatro morenico di Rivoli. Boll. R. Comit. Geol., 18.

[36] SAcco F. (1892). - L'Anfiteadro morenico del Lago Maggiore. Ann. R. Acc. Agric. Torino, 35.

[37] SACCO F. (1893). - Gli Anfiteatri morenici del Lago di Como. Ann. R. Acc. Agric. Torino, 36.

[38] SACCo F. (1895). - L'Anfiteatro morenico del Lago di Garda. Ann. R. Acc. Agric. Torino, 38.
[39] SACCO F. (1898). - Gli Anfiteatri morenici del Veneto. Ann. R. Acc. Agric. Torino, 41.

[40] Sacco F. (1917), - I ghiacciai ed i laghi del Rutor. Boll. Soc. Geol. It., 36.

[41] SACCo F. (1918). - I ghiacciai italiani del gruppo del Monte Bianco. Boll. Com. Glac. It., Is., 3.

[42] SACCO F. (1921-22). - Il Glacialismo nel gruppo del Gran Paradiso. Boll. Com. Glac. It., 1s., 4-5.

[43] SAcco F. (1930), - Il Glacialismo nelle valli Sesia, Strona, Anza e nell'Ossola. Uff. Idrogr. del Po, Pubbl. n. 10.

[44] SACCO F. (1927). - Gli studi glaciologici in Italia. Re lazione e Bibliografia. Cons. Naz. Ricerche, Roma.

[45] PERETTI L. (1930). - I ghiacciai del versante meridionale del Gruppo del Gran Paradiso. Boll. Com. Glac. It., Is., 10.

[46] MONTERIN U. (1922). - Il ghiacciaio di Macugnaga dal 1780 al 1922. Boll. Com. Glac. It., Is., 5.

[47] MONTERIN U. (1923). - Le variazioni secolari del clima del Gran San Bernardo: 1818-1931 e le oscillazioni del Ghiacciaio del Lys al Monte Rosa : 1789-1931. Boll. Com. Glac. It., Is., 12.

[48] MONTERIN U. (1936). - Il clima sulle Alpi ha mutato in epoca storica? Boll. Com. Glac. It., 1s., 16.

[49] Mercial G. (1925). - I ghiacciai del gruppo dell'Adamello. Boll. Comit. Glac. It., 1s., 6.

[50] Mercial G. (1930). - I ghiacciai della Presanella. Boll. Comit. Glac. It., Is., 10.

[51] Desio A. (1967). - I ghiacciai dell'Ortles-Cevedale. 2 vol. CNR-Com. Glac. It.

[52] Somigliana C. (1925). - Sul coefficiente di attrito interno del ghiaccio e la determinazione della profondità dei ghiacciai. Boll. Com. Glac. It., 1s., 6.

[53] Calciati M. (1945). - Le perforazioni eseguite del Ghiacciaio di Hosand. Boll. Com. Glac. It., 1s., 23.

[54] AliverTI G. (1945). - La struttura fisica del ghiacciaio e le modalità del movimento di discesa. Boll. Com. Glac. It., 1s., 23.

[55] UdESCHINI P. (1948). - Moto plastico-viscoso dei ghiacciai. Boll. Com. Glac. It., 1s., 25.

[56] Agostinelui C. (1957-58). - Sul movimento di un ghiacciaio. Nuove ipotesi per la determinazione del profilo di una sezione retta. Boll. Com. Glac. It., 2s., 8.

[57] Giandottr M. (1917). - Studi idrografici sul bacino glaciale del Torrente Lys. Boll. Com. Glac. It., 1s., 2.

[58] ALFIERI S. (1936). - Un quinquennio di osservazioni idrologiche sui torrenti Lys e Rutor. Boll. Com. Glac. It., 1s., 16

[59] AlFIERI S. (1937). - Caratteri idrologici dei maggiori corsi d'acqua piemontesi ed influenza dei ghiacciai sui deflussi. Boll. Com. Glac. It., 1s., 17.

[60] VANNI M. (1957-58). - Il glacialismo attuale nelle Alpi Piemontesi nei rapporti col regime dei corsi d'acqua. Boll. Com. Glac. It., 2s., 8.

[61] VISENTINI M. (1938). - Indagini idrografiche al Ghiacciaio dei Forni (Relazione sullo stato delle ricerche alla fine del 1937). Boll. Com. Glac. It., Is., 18.

[62] ABBADESSA F. (1955), - Rilievi e indagini sul Ghiacciaio dei Forni (Valtellina). Boll. Com. Glac. It., 2s., 6.

[63] COMITATO GLACIOLOGICO ITALIANO (1959-62). Catasto dei ghiacciai italiani. 4 vol. 
[64] CALOI P. (1953). - La prospection séismique appliquée à la détermination de l'épaisseur du glacier (Glacier de la Marmolada). Boll. Com. Glac. It., 2s., 4.

[65] Cassinis R., CARABelli E. (1954). - Misure di spessore del ghiacciaio con metodo sismico al Ghiacciaio dei Forni. Boll. Com. Glac. It., 2s., 5.

[66] AUTORI VARI (1961), - Rilevamenti di ghiacciai e studi glaciologici in occasione dell anno geofisico (Ghiacciaio del Miage). Boll. Com. Glac. It., 2s., 9.

[67] CARABELli E. (1962), - Misure sismiche di spessore del Ghiacciaio del Pian di Neve (Adamello). Boll. Com. Glac. It., 2s., 11.

[68] CARABelli E. (1962), - Esplorazione geofisica al Ghiacciaio del Caresèr. Boll. Com. Glac. It., 2s., 11.

[69] AUTORI VARI (1963), - Rilevamenti di ghiacciai e studi glaciologici in occasione dell'anno geofisico (Ghiacciaio del Calderone). Boll. Com. Glac. It., 2s., 10.

[70] LesCa C., ARMAndo E. (1972), - Determinazioni delle variazioni di superficie e volumetriche dal 1965 al 1970 e controllo della velocità di propagazione delle onde sismiche sul Ghiacciaio de La Lex Blanche. Boll. Com. Glac. It., 2s., 22.

[71] PERETTI L., LESCA C. (1953), - Nuovi rilevamenti topografici di fronti glaciali nella Val Ferret. Boll. Com. Glac. It., 2 s., 4 .

[72] LESCA C. (1974a), - L'espansione della lingua terminale del Ghiacciaio della Brenva in base ai rilievi fotogrammetrici del 1959, 1970 e 1971. Boll. Com. Glac. It., 2s., 20.

[73] LESCA C. (1974b). - Emploi de la photogrammétrie analytique pour la détermination de la vitesse superficielle des glaciers et des profondeurs relatives. Boll. Com. Glac. It., 2s., 22.

[74] COMITATO GLACIOLOGICO ITALIANO (1931). - Rilievi stereofotogrammetrici del Ghiacciaio del Lys. Boll. Com. Glac. It., 1s., 11.

[75] Peretti L., Charrier G. (1964). - Segnalazione ed analisi pollinica di torba alla fronte attuale del Ghiacciaio del Rutor (Valle d'Aosta); considerazioni di paleogeografia e paleoclimatologia locale. Boll. Com. Glac. It., 2s., 14.

[76] BELLONI S. (1970). - Nota preliminare sulle ricerche lichenometriche nell'alveo vallivo del Ghiacciaio dei Forni. Boll. Com. Glac. It., 2s., 18.

[77] ZANON G. (1992), - Venticinque anni di bilancio di massa del Ghiacciaio del Caresèr (Alpi Centrali), 1966-67/199091. Geogr. Fis. Dinam. Quat., 15.

[78] Smiraglia C. (1992). - Sviluppi recenti e prospettive della ricerca glaciologica in Italia. Geogr. Fis. Dinam. Quat., 15.

[79] Belloni S. (1970). - Il bilancio idrologico delle Vedrette del San Giacomo. Boll. Com. Glac. It., 2s., 18.

[80] Belloni S., Catasta G. \& Smiraglia C. (1985). Parametri climatici e variazioni climatiche nel periodo 1950-1982. Geogr. Fis. Dinam. Quat., 8.

[81] CERUTT1 A. (1977), - Variazioni climatiche, alimentazione ed oscillazioni glaciali sul Massiccio del Monte Bianco. Boll. Com. Glac. It., 2s., 8.

[82] Della Ventura A., Rabagliati R., Rampini A., SERANDREI-BARBERO R. (1983). - Remote-sensing ob- servation of glaciers towards their monitoring. 7th Int. Symposium on Remote Sensing of Environment, Ann Arbor, Michigan (May 9-13).

[83] Pantaleo M. (1973), - Bibliografia analitica dei ghiacciai italiani nelle pubblicazioni del C.G.I. Boll. Com. Glac. It., 2s., 21 (suppl.).

[84] SECCHIERI F. (1985). - Problematiche e indicazioni per un nuovo catasto dei ghiacciai. Geogr. Fis. Dinam. Quat., 8.

[85] Rossı G., Tomasıno M. (1992), - Impiego dei sistemi informativi territoriali (S.I.T.) nella valutazione della producibilità idroelettrica e della sensitività climatica dei bacini nivo-glaciali. Geogr. Fis. Dinam. Quat., 15.

[86] GiAda M., ZANON G. (1991). - Variazioni di livello $e$ volumetriche sulla Vedretta del Caresèr (Gruppo OrtlesCevedale) tra il 1980 et il 1990. Geogr. Fis. Dinam. Quat., 14.

[87] Rossı G.C., Mercalli L., SPANNA F. (1993). - Recenti ricerche sui ghiacciai italiani del Monte Rosa : primi risultati. Nimbus 1.

[88] Mercalli L., Reynaud L., Vincent C. (1993), - Mesures de bilan de masse 1991-92, Glacier du Ciardoney. Massif du Grand Paradis, Italie. Soc. Hydr. de France, Sect. Glac., Grenoble, mars 1993.

[89] Catasta G., Smiraglia C. (1992). - The mass balance of a cirque glacier in the Italian Alps (Ghiacciaio della Sforzellina, Ortles-Cevedale Group). J. of Glac., 131.

[90] SERVIZIO GLACIOLOGICO LOMBARDO (1992). Ghiacciai in Lombardia. Ed. Bolis, Bergamo.

[91] Dutto F., Godone F., Mortara G. (1991). - L'écroulement du Glacier Supérieur de Coolidge (paroi nord du Mont Viso, Alpes occidentales). Revue Géogr. Alpine, 30.

[92] Dutto F., MORTARA G. (1992). - Rischi connessi con la dinamica glaciale nelle Alpi Italiane. Geogr. Fis. Dinam. Quat., 15.

[93] Resnati C., Smiraglia C. (1990). - Determinazione della struttura interna del Rock Glacier di Val Pisella (Alta Valtellina) attraverso sondaggi elettrici verticali. Risultati e problemi. Geogr. Fis. Dinam. Quat., 13.

[94] Pelfini M., Smiraglia C. (1992). - Recent fluctuations of glaciers in Valtellina (Italian Alps) and climatic variations. J. of Glac., 129.

[95] Mazza A., Mercall.1 L. (1992). - Il Ghiacciaio Meridionale dell'Hohsand (Val Formazza) : un secolo di evoluzione climatica e rapporti con la produzione idroelettrica. Oscellana, 22.

[96] Orombelli G., Smiraglia G., Terranova R. (1994), Verso una nuova geografia delle terre polari : sintesi e prospettive. Atti Conv, Roma, 21-22 nov. 1991. Mem. Soc. Geogr. It., 51

[97] OROMBELLI G. (1991). - Le ricerche glaciologiche nell'ambito del « Programma Nazionale di Ricerche in Antartide $":$ risultati e prospettive. Geogr. Fis. Dinam. Quat., 15.

[98] ZANON G. (1990), - I ghiacciai del Veneto. Giunta Regionale del Veneto, Ed. Canova.

[99] MERCALlı L. (a cura di) (1991). - La montagna di ghiaccio. Storia dei ghiacciai italiani del Monte Rosa. CGI, Torino.

[100] Smiraglia C. (1992). - Guida ai ghiacciai e alla glaciologia. Ed. Zanichelli, Bologna. 